\title{
Exéresis de quiste branquial lateral: presentación de un caso manejado con solución electrolizada de superoxidación con $\mathrm{pH}$ neutro
}

\author{
Excision of a lateral branchial cyst: case report \\ treated with neutral electrolyzed water
}

\author{
Juan Paz García,* Félix Manuel Hernández Alemán, Herminia Farías López*
}

\section{RESUMEN}

Entre los quistes cervicales congénitos, los quistes branquiales son los segundos más frecuentes después de los quistes tiroglosos, alcanzando $30 \%$ de incidencia. Los quistes branquiales son patologías raras con baja incidencia, aunque se les considera la alteración congénita más común de la región cervical. Su etiología no está bien establecida, pero se asocia con errores del desarrollo embrionario. Dependiendo del arco branquial en que se ubiquen, se clasifican en tipos del I al IV. El 90-95\% de los casos corresponden al tipo II, derivado del segundo arco branquial y localizados en el tercio superior del borde anterior del músculo esternocleidomastoideo, en la cara lateral del cuello. En general, estas masas benignas son indoloras, pero pueden aumentar rápidamente su tamaño, limitando el movimiento cervical, e incluso provocar disfagia o compromiso respiratorio; además, siempre existe riesgo latente de infección. Su diagnóstico diferencial se realiza con exploración, ecografías, aspiración con aguja fina e histopatología. El tratamiento consiste en la extirpación completa del quiste, pudiendo

\section{ABSTRACT}

Brachial cysts are rare pathologies with a low incidence, although they are considered the most common congenital disorder of the cervical region. Its etiology is not well established, but it is associated with errors in embryonic development. Depending on the branchial arch in which they are located, they are classified into types I-IV. Between $90-95 \%$ of cases correspond to type II, derived from the second branchial arch, and located in the upper third of the anterior border of the sternocleidomastoid muscle, on the lateral aspect of the neck. These benign masses are generally painless but can rapidly increase in size by limiting cervical movement and even causing dysphagia or respiratory compromise, and there is always a latent risk of infection. Its differential diagnosis is made with exploration, ultrasound, fine needle aspiration and histopathology. Treatment consists of complete removal of the cyst, which can be challenging due to the proximity of neurovascular structures. In this opportunity, we present our experience in the surgical approach of a type II brachial cyst, located in the left cervical lateral region,

* Centro Hospitalario Unión. Colima, México.

‡ Hospital Ángeles Culiacán. Sinaloa, México.

\author{
Correspondencia: \\ Juan Paz García \\ E-mail: dr_juanpaz@hotmail.com
}

Citar como: Paz GJ, Hernández AFM, Farías LH. Exéresis de quiste branquial lateral: presentación de un caso manejado con solución electrolizada de superoxidación con pH neutro. Rev Mex Cir Bucal Maxilofac. 2021;17 (1): 28-33. https://dx.doi.org/10.35366/100258 
ser un desafío debido a la cercanía de estructuras neurovasculares. El presente trabajo muestra el abordaje quirúrgico de un quiste branquial tipo II, en la región lateral cervical izquierda y la notable recuperación de la herida quirúrgica, lograda con el uso de solución electrolizada de superoxidación con $\mathrm{pH}$ neutro (SES), en el lavado del lecho quirúrgico, para minimizar el riesgo de infecciones postoperatorias y favorecer la recuperación de la herida.

Palabras clave: Quiste branquial lateral, tipo II, solución electrolizada de superoxidación con $\mathrm{pH}$ neutro (SES). and the remarkable evolution of the wound healing, due to the use of neutral electrolyzed water (SES) for the lavage or the surgical bed; in order to minimize the risk of postoperative infections and promote the healing of the surgical wound.

Keywords: Branchial lateral cyst, type II, neutral electrolyzed water (SES).

\section{INTRODUCCIÓN}

Los remanentes de la oclusión incompleta de los arcos, hendiduras y bolsas branquiales durante el desarrollo embriológico pueden dar lugar a la aparición de un nódulo o masa en el cuello, entre el músculo esternocleidomastoideo y la faringe, denominado quiste branquial. ${ }^{1}$ Su origen también puede deberse a restos del seno cervical, de conductos tirofaríngeos o a la inserción de epitelio escamoso en un ganglio linfático cervical. ${ }^{1} \mathrm{El}$ aparato branquial consiste en seis pares de arcos que rodean la faringe; las deformidades en el primer par corresponden cerca de $1 \%$ de los casos de los quistes branquiales, mientras que aquellas que se localizan en el segundo par son las más frecuentes, con 90 a $95 \%$ de los casos; en tanto que quistes en el tercer o cuarto arco son muy raros. ${ }^{2}$

Bailey clasificó a los quistes branquiales del segundo arco branquial en cuatro subtipos; según su ubicación entre la amígdala palatina y el borde anterior del músculo esternocleidomastoideo, en la región lateral y su relación con la vaina carotídea. ${ }^{1}$ Los del tipo I tienen una localización superficial a lo largo del borde anterior del músculo esternocleidomastoideo debajo de la fascia cervical, sin contactar la vaina carotídea; los quistes del tipo II son adyacentes a la vaina carotídea, mientras que los del tipo III se ubican entre la carótida interna y externa, y los del tipo IV se presentan en el espacio de la mucosa faríngea y medial a la vaina carotídea contactando con la pared faríngea a nivel de la fosa tonsilar. ${ }^{1,2}$

Los signos clínicos de estas anormalidades son la presencia de una masa unilateral cervical, al tacto lisa, redondeada y móvil que suelen aparecer en las primeras décadas de la vida. En general son benignas, indoloras y no limitantes en la vida del paciente; sin embargo, pueden aumentar de tamaño, generar complicaciones y existe el riesgo de malignización. ${ }^{3}$
Su volumen se incrementa debido a hemorragias intraquísticas o infecciones en las vías aéreas superiores. ${ }^{2}$ Un quiste con magnitud incrementada puede generar dificultad del movimiento cervical, torticolis y en casos extremos, aunque poco frecuentes, disfagia o disnea. ${ }^{4}$ Su diagnóstico se realiza mediante la exploración, técnicas radiológicas o ecografías y mediante la técnica de aspiración con aguja fina; si en el estudio citológico del aspirado se encuentran células epiteliales benignas y hay presencia de cristales de colesterol en una secreción mucoide fluida es presuntivo de un quiste branquial. ${ }^{2,5}$

El único tratamiento para este padecimiento es la escisión quirúrgica completa del quiste. No obstante, el procedimiento puede ser un reto debido a la cercanía de las estructuras neurovasculares tales como la arteria carótida, la vena yugular interna, la rama marginal mandibular del nervio facial, el gran nervio auricular y los nervios vago, accesorio e hipogloso. ${ }^{5-7}$ Las complicaciones en este procedimiento son bajas, pero una escisión incompleta del quiste puede resultar en recurrencia, la cual puede ser de $3 \%$ en la primera intervención e incrementarse hasta $22 \%$ en intervenciones subsecuentes. ${ }^{3,8}$

En este trabajo se describe el manejo quirúrgico realizado sobre un quiste branquial lateral tipo II $y$, particularmente, el efecto de utilizar solución electrolizada de superoxidación con pH neutro (SES), como irrigante del lecho quirúrgico, en el transoperatorio, y de gel SES como apósito protector antiséptico y adyuvante de hemostasia y regeneración tisular sobre la herida quirúrgica. Previamente establecimos que los lavados con SES durante un procedimiento quirúrgico tienen efecto adyuvante en la hemostasia, además de controlar la respuesta inflamatoria y la carga bacteriana, lo cual ha sido corroborado en otros estudios, específicamente en casos de sepsis abdominal, en los cuales también se ha observado que su uso disminuye el tiempo 
de estancia hospitalaria. ${ }^{9-11}$ Asimismo, se estableció que las SES inducen regeneración tisular, efecto que igualmente ha sido ratificado en quemaduras y úlceras de pie diabético. ${ }^{9,12,13}$ Por estas propiedades, se decidió utilizar SES y gel SES durante la exéresis de un quiste branquial.

\section{DESCRIPCIÓN DEL CASO}

En 2014 se presentó paciente femenino de 49 años de edad en una clínica particular de la ciudad de Colima, Colima, quien mostraba un aumento visible de la región cervical izquierda (Figura 1A-B). Manifestó tener evolución de varios años, aunque no precisó el tiempo exacto; sin embargo, la paciente dijo tener dos semanas con molestias al giro cervical. Se procedió a palpar la zona cervical izquierda, con aumento de volumen ocupando espacio submandibular por delante del músculo esternocleidomastoideo, sin aparente infartación de cadenas ganglionares cervicales y sin datos de parestesia.

Se observaron exámenes de laboratorio preoperatorios sin alteraciones aparentes, además se indicó un estudio de ultrasonido, por considerarlo estudio de elección, ya que arroja datos precisos y mediciones sin el uso de radiación. La ecografía mostró una lesión de $5.44 \times 2.37 \mathrm{~cm}$ con contenido líquido, localizada por delante del músculo esternocleidomastoideo y posterior a la glándula submaxilar (Figura 1C). Por estas características se diagnosticó un quiste branquial lateral tipo II, de acuerdo con la clasificación de Bailey.

Se procedió, bajo anestesia general por intubación orotraqueal, a realizar exéresis con abordaje cervical en línea de tensión o de Langer (Figura 2A-D). Se disecó cuidadosamente la pared medial-secretora del quiste, que orienta y da seguridad quirúrgica para no lesionar estructuras vitales, lavando profusamente con solución electrolizada de superoxidación con $\mathrm{pH}$ neutro y especies activas de cloro y oxígeno al $0.002 \%$, SES (Estericide ${ }^{\circledR}$ Antiséptico Irrigación Quirúrgica). De esta manera, se obtuvo una buena visión del lecho quirúrgico, lo cual disminuyó el riesgo de perforaciones a la pared o de daño a estructuras vasculares o nerviosas involucradas en el trayecto y grandes abordajes. De modo subsecuente, se realizó la enucleación total de la cápsula de la pared medial secretora, efectuando abundante lavado con SES, hasta obtener solución límpida. Por último, el lecho quirúrgico se rellenó con gel SES (gel a base de solución electrolizada de superoxidación de amplio espectro, Oxoral ${ }^{\circledR}$ gel antiséptico) (Figura 3A-B); se dejó drenaje Penrose de $1 / 4$ y puntos simples con sutura nylon 4-0 y aguja traumática. Se colocó apósito recolector y se indicó medicación postoperatoria, antiinflamatorios no esteroideos y antibióticos: naproxeno $500 \mathrm{mg}$ oral por cinco días y ciprofloxacino $500 \mathrm{mg}$, oral por siete días (Figura 3C). Después de 48 horas se retiró el drenaje, dando alta hospitalaria por mejoría y los puntos de sutura se retiraron a los siete días de la intervención (Figura 3D).

Cabe mencionar que el espécimen retirado contenía un líquido amarillento pastoso (Figura 2B) y el análisis histopatológico mostró que el tejido extirpado estaba formado por una cápsula epitelial con tejido poliestratificado plano con varias capas de células paraqueratinizadas con presencia de células mucosas y cristales de colesterol; lo que corroboró el diagnóstico clínico y de imagen. A cinco años de seguimiento no hay indicios de recidiva.
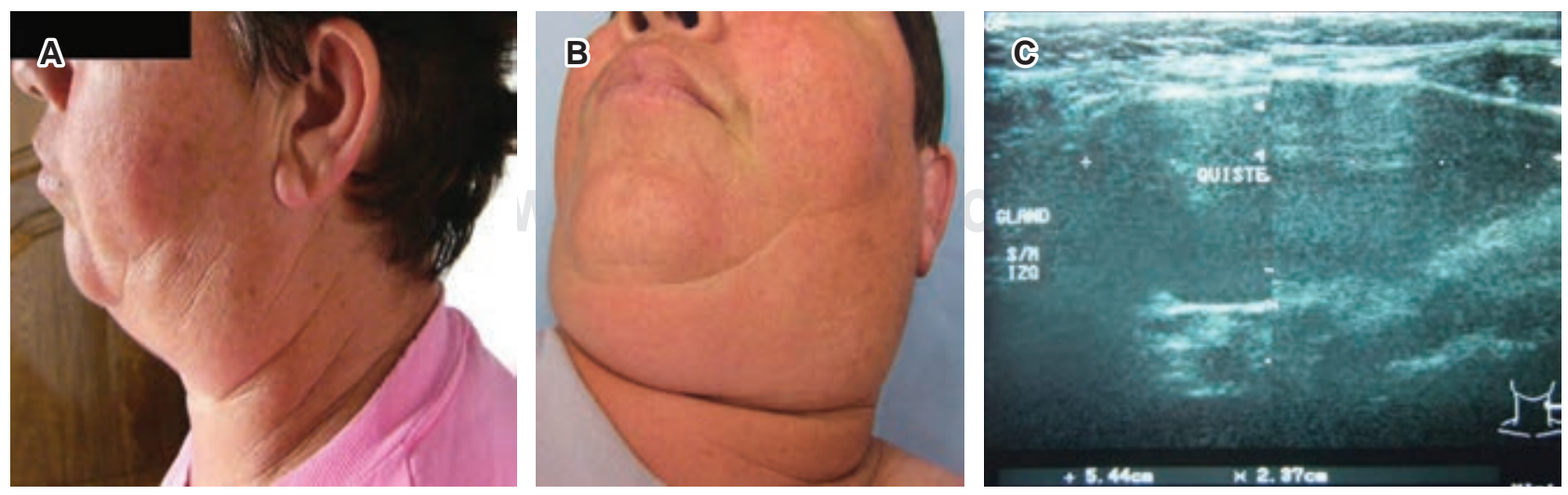

Figura 1: A) Vista de perfil del aumento del volumen en la región cervical lateral izquierda. B) Vista inferior. C) Imagen de ultrasonido en la que se aprecia una masa de $5.44 \times 2.37 \mathrm{~cm}$ con contenido líquido. 

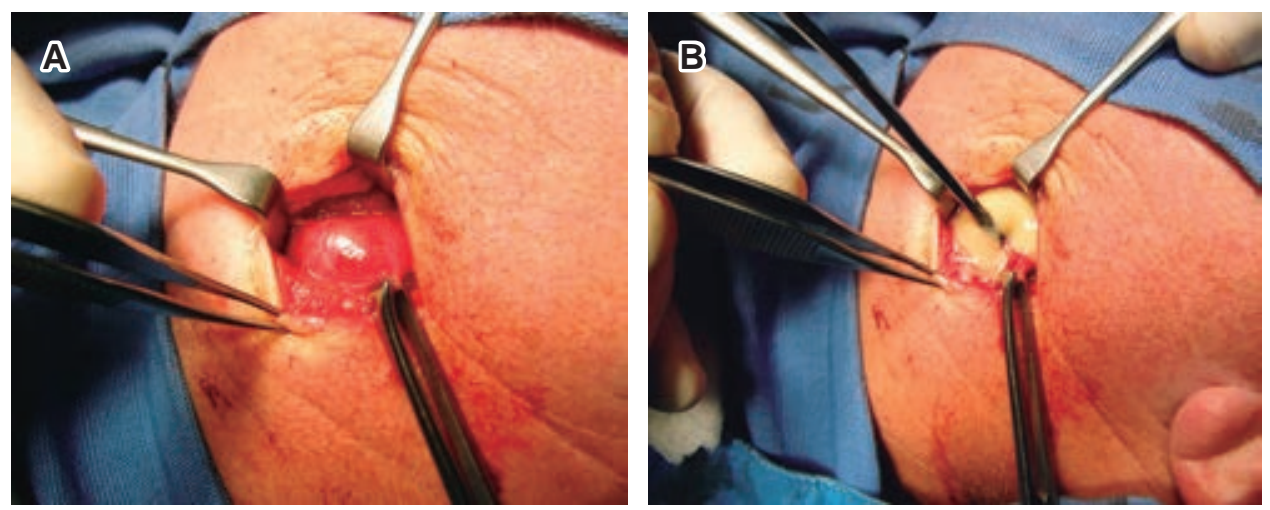

Figura 2:

A-C) Realización del proceso de exéresis con abordaje cervical en línea de tensión. B) Líquido amarillento pastoso al interior del quiste. C) Remoción del

quiste. D) Aspecto del espécimen removido.
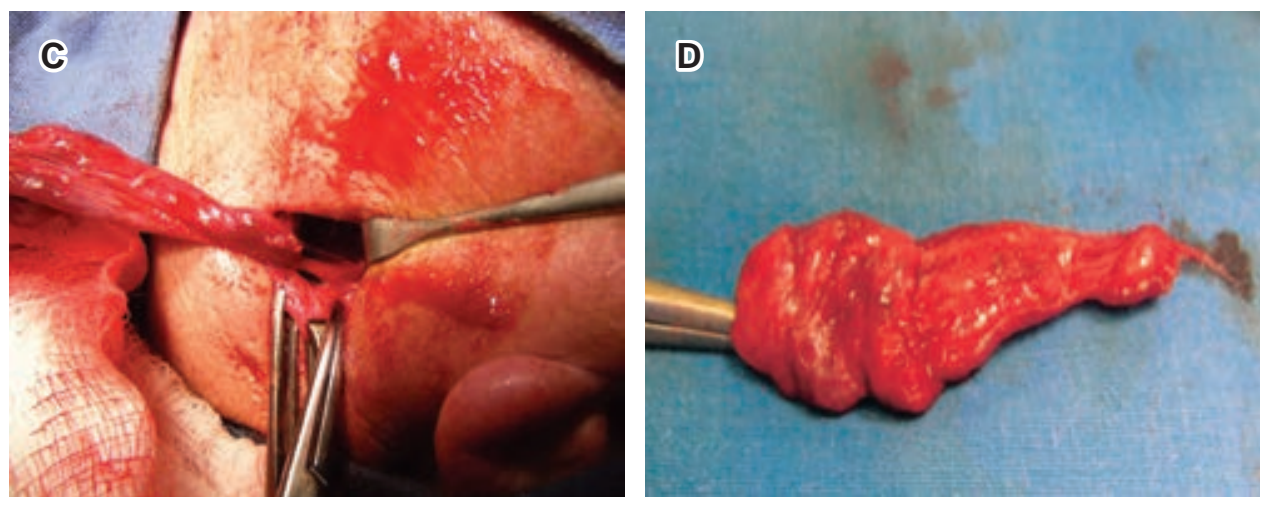
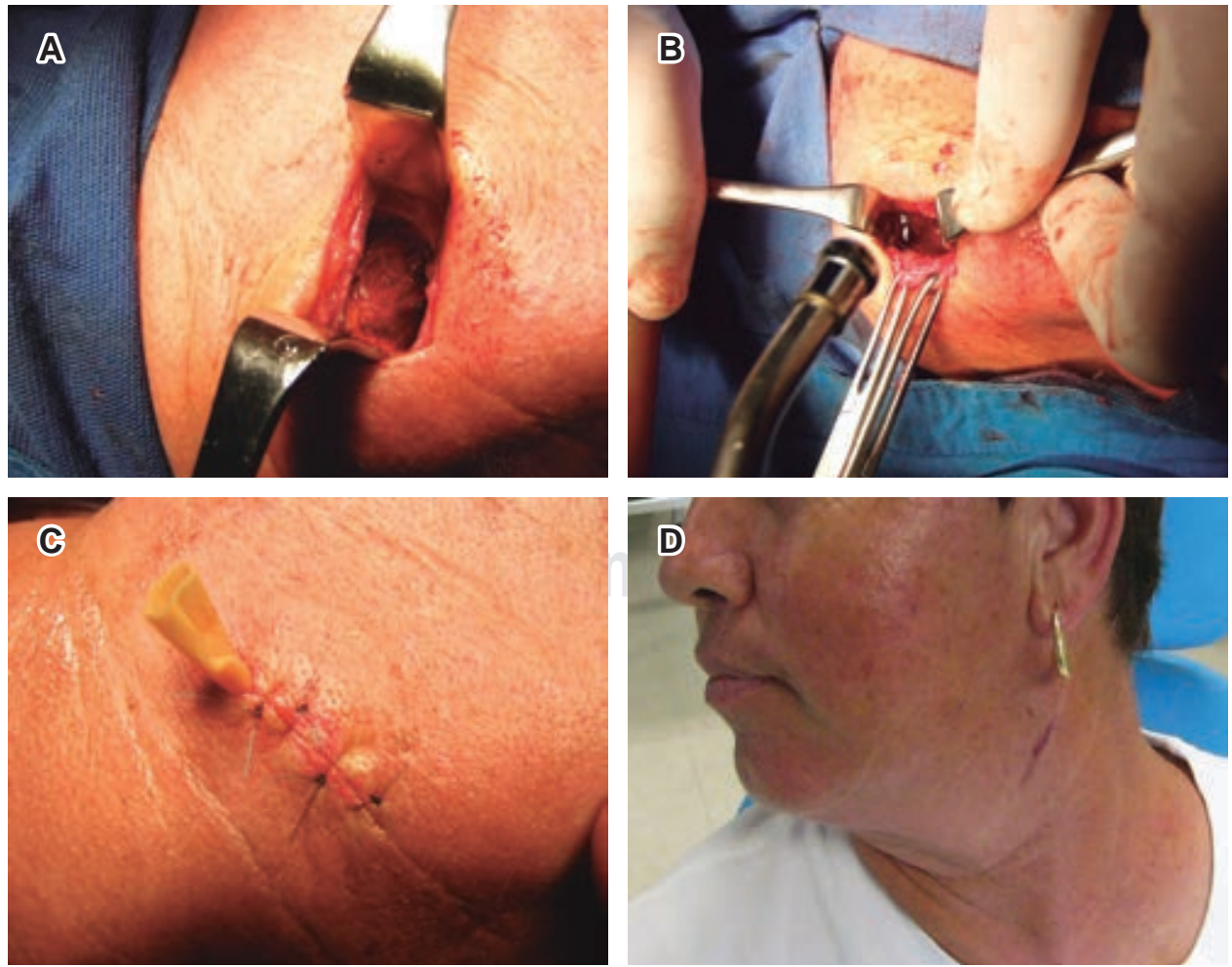

Figura 3:

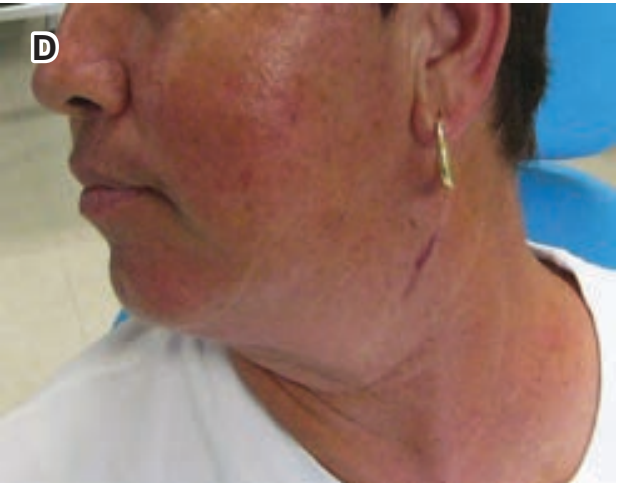

A) Postenucleación de la cápsula, el lecho quirúrgico fue lavado profusamente con solución electrolizada de superoxidación con $\mathrm{pH}$ neutro (SES). B) La cavidad se rellenó con gel SES. C) Se dejó drenaje Penrose que se retiró 48 horas después. D) Aspecto de la zona cervical lateral izquierda, siete días posteriores a la cirugía. 


\section{DISCUSIÓN}

El tratamiento de los quistes branquiales, es decir, su escisión completa, puede ser un reto para el cirujano debido a la cercanía de estructuras neurovasculares, como la vena yugular interna a la que el quiste suele estar adherido. ${ }^{5}$ Por lo tanto, las complicaciones más comunes de este procedimiento pueden ser entumecimiento y parestesia, ya que depende de la habilidad del cirujano para retirar completamente la masa sin dañar las estructuras adyacentes. Otras complicaciones son el desarrollo de seromas, hematomas e infecciones en la herida, así como una cicatrización hipertrófica. ${ }^{8}$ La reincidencia en estos casos es baja, entre 3 y $4 \%$; sin embargo, si el quiste no es completamente retirado en la primera intervención, el índice de reincidencia incrementa hasta en $20 \%{ }^{8}$ Un diagnóstico equivocado y en consecuencia un tratamiento erróneo son las causas de que el quiste no sea retirado en su totalidad. Deben diferenciarse, por consiguiente, de un absceso cervical, un ganglio linfático neoplásico o un absceso frío. Asimismo, es importante no romper el quiste durante el proceso quirúrgico, pues, aunque el riego es bajo, existe el potencial de malignización. ${ }^{5}$

En este reporte clínico se muestra el abordaje quirúrgico realizado para retirar una masa que se localizaba en el área cervical lateral izquierda de una paciente, limitando su movimiento del cuello. Los estudios de imagenología mostraron un quiste de dimensiones considerables, ubicado por delante del músculo esternocleidomastoideo y posterior a la glándula submaxilar, por lo que se diagnosticó un quiste branquial tipo II, posteriormente confirmado por histopatología.

Durante la exéresis, se decidió utilizar solución electrolizada de superoxidación de pH neutro (SES). La justificación fue que previamente observamos y reportamos que el lavado con este tipo de soluciones tiene un efecto adyuvante de la hemostasis durante las intervenciones quirúrgicas. ${ }^{9}$ Es importante recordar que estos quistes contienen doble pared, lo que facilita al cirujano la enucleación de la pared medial secretora, y con ausencia de sangrado permite tener un campo de visión limpio durante la cirugía sin el riesgo de daño a los tejidos. Esto permite minimizar el riesgo de lesionar otras estructuras, en este caso, particularmente las venas y nervios cervicales, lo cual reduce el riesgo de complicaciones como la parestesia.

Por otro lado, el uso de SES antiséptico como irrigante del lecho quirúrgico permite el arrastre de posibles contaminantes como patógenos, así como su inactivación, lo que estrecha las posibilidades de infecciones postquirúrgicas. ${ }^{10,11}$ Otra ventaja del lavado de cavidades con SES es el control de los procesos de la respuesta inmunitaria generados por el trauma de la cirugía y la modulación que ejerce hacia la reparación de tejidos que finalmente llevan a una rápida reparación tisular, disminuyendo los tiempos de estancia hospitalaria. ${ }^{10,11} \mathrm{Si}$ bien los mecanismos exactos por los cuales las SES tienen estos efectos no se han establecido, las especies reactivas de las soluciones electrolizadas de superoxidación de $\mathrm{pH}$ neutro podrían estar regulando la producción de citocinas antiinflamatorias, contrarrestando el ambiente proinflamatorio y enfocando la respuesta a la reparación de tejidos a través del incremento en la producción de IL-10, quimiotácticos de macrófagos, factores de crecimiento como TGF- $\beta$ e induciendo la proliferación y migración de queratinocitos, así como la diferenciación de fibroblastos. ${ }^{14-18}$

El uso de la SES para lavados y la aplicación del gel SES en el lecho quirúrgico resultante de la exéresis del quiste branquial, efectivamente nos permitió tener un campo de visión limpio durante el procedimiento quirúrgico; además de que asociamos su uso con la ausencia de complicaciones postoperatorias, como infecciones, hematomas o seromas y con la pronta recuperación de la herida, sin signos de hipertrofia.

\section{CONCLUSIONES}

La exéresis de un quiste branquial lateral tipo II, según la clasificación de Bailey, fue realizada de manera exitosa sin complicaciones postoperatorias y con una recuperación y cicatrización normal, lo cual asociamos al uso de soluciones electrolizadas de superoxidación con $\mathrm{pH}$ neutro durante el proceso quirúrgico y en la etapa de recuperación. Se postula su uso como agente útil para la irrigación o el lavado del lecho quirúrgico, pues favorece claridad en el campo quirúrgico, controla la inflamación, refuerza la antisepsia del campo quirúrgico e induce recuperación acelerada de la herida quirúrgica.

\section{AGRADECIMIENTOS}

A la empresa Esteripharma SA de CV, por proporcionar la solución electrolizada de superoxidación con $\mathrm{pH}$ neutro y el gel SES, utilizados en la resolución del caso presentado. 


\section{REFERENCIAS}

1. Abdelfattah HM, Ahmed ME, Ahmed Mel-R, Ahmed MA, Moussa $A E$. A branchial cyst of the pyriform fossa transoral laser resection: a case report. Eur Arch Otorhinolaryngol. 2016; 273 (2): 525-528.

2. Veloz T Marcela, Pacheco TA. Presentación de quiste de segundo arco branquial como masa orofaríngea: Reporte de un caso. Rev Otorrinolaringol Cir Cabeza Cuello. 2015; 75 (2): 157-160.

3. Altuna-Mariezkurrena X, Luqui-Albisua I, Vea-Orte JC, AlgabaGuimerá J, Echenique-Elizondo M. Quiste branquial. A propósito de dos casos. Gac Med Bilbao. 2005; 102 (4): 92-94.

4. Vargas Domínguez R. Quiste branquial. Reporte de tres casos. Odontol Actual. 2008; 5 (59): 20-23.

5. Slater J, Serpell JW, Woodruff S, Grodski S. Role of fine needle aspiration cytology in the preoperative investigation of branchial cysts. ANZ J Surg. 2012; 82 (1-2): 42-45.

6. Castro Pérez F, Rodríguez González R, Flores Contreras JM, Álvarez Díaz V, Cordero Ledesma MN. Quiste branquial tipo I: presentación de un caso. Rev Ciencias Médicas. 2010; 14 (4): 108118.

7. Kotecha V, Muturi A, Ruturi J. Branchial cysts: an unusual cause of a mediastinal mass: a case report. J Med Case Rep. 2015; 9: 208.

8. Daoud FS. Branchial cyst: an often forgotten diagnosis. Asian J Surg. 2005; 28 (3): 174-178.

9. García JP, Maldonado RA, Díaz RI, Muñiz J, Rodríguez HA. Sustitución del uso de solución salina fisiológica como irrigante en el manejo de pacientes sépticos y quirúrgicos por solución electrolizada. Rev Mex Cir Bucal Maxilofac. 2011; 7 (2): 46-52.

10. Singal R, Dhar S, Zaman M, Singh B, Singh V, Sethi S. Comparative evaluation of intra-operative peritoneal lavage with super oxidized solution and normal saline in peritonitis cases; randomized controlled trial. Maedica (Bucur). 2016; 11 (4): 277-285.

11. Nachón GFJ, Díaz TJ, Benítez OF, García GF, Santiago GJ, Martínez CAJ. Lavado peritoneal transoperatorio con solución electrolizada por selectividad iónica en peritonitis secundaria. Cir Gen. 2010; 32 (1): 11-16.

12. Nakae $\mathrm{H}$, Inaba $\mathrm{H}$. Electrolyzed strong acid aqueous solution irrigation promotes wound healing in a burn wound model. Artif Organs. 2000; 24 (7): 544-546.

13. Assaloni MD, DaRos R. Super-oxidized solution (SOS) therapy for infected diabetic foot ulcers. Wounds. 2006; 18 (9): 262-270.

14. Bocci V. How a calculated oxidative stress can yield multiple therapeutic effects. Free Radic Res. 2012; 46 (9): 1068-1075.

15. Schieber M, Chandel NS. ROS function in redox signaling and oxidative stress. Curr Biol. 2014; 24 (10): R453-R462.

16. Holmstrom KM, Finkel T. Cellular mechanisms and physiological consequences of redox-dependent signalling. Nat Rev Mol Cell Biol. 2014; 15 (6): 411-421.

17. Finkel T. Signal transduction by reactive oxygen species. J Cell Biol. 2011; 194 (1): 7-15.

18. Diwanji N, Bergmann A. An unexpected friend - ROS in apoptosisinduced compensatory proliferation: Implications for regeneration and cancer. Semin Cell Dev Biol. 2018; 80: 74-82. 